\title{
Application of Enhanced Recovery after Surgery in Gastrointestinal Surgery based on Multidisciplinary Team
}

\author{
Dan Liu ${ }^{1}$ \\ ${ }^{1}$ Department of Gastrointestinal Surgery of Affiliated Hospital of Qingdao University, China
}

\begin{abstract}
Chinese gastrointestinal surgeons are building up our own high level evidence in evidence-based medicine, With tremendous clinical trials carrying out in gastrointestinal cancers. Besides, Standardized procedure of diagnosis and treatment should be promoted. More personalized schemes are needed. The model of multidisciplinary team can be more widely and deeply applied. In order to further optimize the surgical pathways and promote the clinical application of ERAS in surgery, it is important to raise awareness of the ERAS concept, advocate multi-disciplinary cooperation model, including surgery, anesthesiology, nursing, clinical nutrition, etc. to improve the application of ERAS in gastrointestinal surgery, promote medical and health management performance. And the concept of enhanced recovery after surgery should be more operable. To keep pace with the times, the gastrointestinal surgeons have to seek for innovative technology and new ideas.
\end{abstract}

Keywords: ERAS, Multidisciplinary Team, Gastrointestinal Surgery, Anaesthesia

Enhanced recovery after surgery (ERAS) is a multidisciplinary protocol of care delivered to patients with the aim of maintaining normal physiology and thereby facilitating postoperative recovery ${ }^{1}$. Based the original work of Kehlet and Wilmore ${ }^{2}$, the ERAS protocol is now used routinely, especially in the field of surgery for colorectal cancer. The combination of various perioperative approaches has led to significant reductions in surgical morbidity and mortality rates, the duration of hospital stay, and the cost of hospitalization, and has greatly improved postoperative recovery ${ }^{3}$.

The main contents of ERAS perioperative management include: patients education, optimization of anesthetic prescription, reduction of operative stress, control of nausea and vomiting and postoperative intestinal paralysis, management of multi-mode analgesia, management of catheter, reasonable nutritional support , and standards and planning of discharge ${ }^{1}$. ERAS is a systematic project designed for preoperative and postoperative multistage management. Therefore, ERAS in multidisciplinary collaboration mode has been widely recognized.

\section{Application of ERAS in gastrointestinal surgery}

\section{1 Preparation before operation}

\subsubsection{Preoperative education and communication}

The good communication with patients, can improve the negative emotion and enhance the self-confidence of the patients and the family, and can obviously reduce the anxiety of the patients and improve patients' postoperative recovery; on the other hand, the patient's trust can be obtained, and the ideal doctor-patient relationship can be established.

\subsubsection{Evaluation and control of basic diseases}

The majority of gastric cancer patients are elderly, who are always accompanied by hypertension, diabetes, heart failure, chronic obstructive pulmonary disease, anemia, hypoproteinemia or malnutrition. These complications seriously affect the clinical outcome of patients. Before operation, the patients' cardiopulmonary function should be evaluated actively, and be given symptomatic treatment in time, for adjusting the whole body state of the patients. Patients should be educated to give up smoking and drinking, exercise the cardiopulmonary function, and improve the state of malnutrition.

\subsubsection{Gastrointestinal preparation}

Studies have shown that traditional methods (fasting at night before operation, cleaning enema, etc.) do not reduce the incidence of postoperative complications, but can make intestinal wall edema and brittleness prone to bleeding. Repeated mechanical enema and diarrhea also affected patients' rest and reduced their physical fitness. It has been agreed that there is no need to clean the intestine before gastrointestinal surgery, and less recommendation of oral osmotic laxatives and clean enema, which helps to maintain water and electrolyte acid-base balance ${ }^{4}$.

\subsubsection{Oral carbohydrate before operation}

Long-term fasting may decrease preoperative energy

This article is published under the terms of the Creative Commons Attribution License 4.0

Author(s) retain the copyright of this article. Publication rights with Alkhaer Publications.

Published at: http://www.ijsciences.com/pub/issue/2019-02/

DOI: 10.18483/ijSci.1920; Online ISSN: 2305-3925; Print ISSN: 2410-4477 
reserve and lead to intraoperative hypoglycemia and starvation stress. Since liquid food can be emptied after 2 hours, it is suggested that oral $50 \mathrm{~g}$ of carbohydrates 2 hours before anesthesia can relieve anxiety and hunger caused by fasting before anesthesia, reduce the degree of insulin resistance after operation, and do not increase the risk of accidental aspiration during anesthesia ${ }^{5}$.

\subsubsection{Prophylactic use of antibiotics}

Patients undergoing major abdominal surgery should be given prophylactic antibiotics half an hour before operation. If the operation time is longer than 3 hours, antibiotics should be added once.

\subsection{Intraoperative measures}

\subsubsection{Operation safety management}

Surgical safety verification system can significantly reduce the incidence and mortality of surgical complications, improve the clinical outcome of patients, and recommend the application in the global operation process $^{6}$. When the patient enters the operating room, before the operation begins and leaves the operating room, must strictly carry out the check system, prevents the wrong operation patient, the wrong operation place and the wrong operation. Safety check before operation can avoid all kinds of harmful risks of elective operation, which is of great significance to promote communication and communication between operation teams and prevent surgical mistakes.

\subsubsection{Anesthesia management}

General anesthesia can delay the recovery of gastrointestinal function after operation. Thoracic epidural block combined with general anesthesia can reduce the dose of anesthetic, reduce the effect on gastrointestinal function, and effectively inhibit the stress response of gastric cancer patients during perioperative period $^{7}$. It also can maintain hemodynamics stable and is beneficial to postoperative analgesia and shortening the time of postoperative intestinal paralysis. General anaesthesia drugs should be selected for quick, short-acting anesthetics or short-term opiates such as fentanyl, which can ensure that patients can wake up quickly after surgery and reduce the residual effect and adverse reactions of anesthetic drugs.

\subsubsection{Liquid management}

The application of goal-oriented blood pressure control and infusion theory can ensure the blood perfusion of important organs. The blood pressure should be properly controlled at a lower level, then adjusted to a normal level after operation, and the volume of fluid should be strictly controlled, in order to avoid tissue edema caused by water overload ${ }^{8}$.

\subsubsection{Avoid hypothermia during operation}

During the operation, the adaptation ability of gastric cancer patients to environmental changes was decreased, a large amount of hypothermia fluid was injected. Using unheated flushing fluid and the inhibitory effect of general anesthesia on the center of body temperature regulation were easy to cause hypothermia and aggravate the stress response, which lead to abnormal coagulation function, even induce arrhythmia, and prolong anesthesia recovery time ${ }^{9,10}$. Therefore, it is necessary to keep operating room and liquid temperature suitable, and body temperature should be monitored and thermal blanket should be used to prevent adverse reactions caused by hypothermia. Antithrombotic socks and mechanical antithrombotic pump should be used to prevent deep venous thrombosis ${ }^{1}$.

\subsubsection{Surgical mode selection}

The concept of minimally invasive surgery advocated by ERAS should be applied throughout the operation of gastric cancer, and the surgical incision should be minimized on the basis of the full exposure of the surgical field. Avoid injury and bleeding, minimize blood transfusion and shorten operation time. At present, more than $60 \%$ of patients with gastric cancer in Japan and South Korea are early pathological changes, and laparoscopic surgery is also recommended, which can reduce surgical trauma stress, promote patients' recovery, and quality of life. The KLASS (Korea) and CLASS (China) studies on laparoscopic radical gastrectomy for advanced gastric cancer have shown excellent surgical safety and short-term tumor control effects. The effect is comparable to that of previous open surgery ${ }^{11}$.

\subsubsection{Tube management}

Because various drainage tubes can obviously affect the postoperative activity and increase the negative psychology of the patients, it is suggested that the indications for the use of all kinds of catheters should be strictly grasped. Previous studies have shown that it is safe for patients with gastric cancer not to use nasogastric tube during selective abdominal surgery ${ }^{12}$. Placing nasogastric tube for a long time after operation can increase the risk of oropharyngeal side effects and pulmonary infection. Urinary catheter should be removed 24 hours after operation to avoid discomfort or urinary tract infection. Although RCT studies have shown that conventional use of drainage tubes in cholecystectomy, joint replacement, colectomy, thyroidectomy and hysterectomy, and pancreatectomy is not beneficial. However, the need for abdominal drainage after radical gastrectomy is still controversial. There is still a risk of anastomotic leakage, bleeding or lymphatic leakage after radical gastrectomy, so it is necessary to place a peritoneal drainage tube.

\subsection{Postoperative management}

\subsubsection{General treatment}

ERAS encourages patients to get out of bed early after operation, which can reduce the loss of muscle tissue, improve lung function and oxygen cooperation, promote postoperative intestinal peristalsis and prevent deep venous thrombosis of lower extremity. The 
amount of fluid should be restricted properly, and $2000 \mathrm{ml}$ should be taken as the best one every day. Too much liquid could cause tissue edema, delay the recovery of gastrointestinal function, and reduce the healing ability of anastomotic stoma. Restrictive fluid infusion can reduce edema, increase serum albumin content and reduce the incidence of postoperative complications 9 . The traditional view is that flatus and defecate is the standard for the recovery of intestinal function. Therefore, gastric cancer surgery needs to keep patients from eating for a long time. The concept of ERAS suggests that colorectal surgery patients can eat before their gastrointestinal function is fully recovered. Early enteral nutrition could promote intestinal peristalsis, decrease catabolism, protect intestinal mucosal barrier and enhance postoperative immune function ${ }^{13}$. But it is necessary to carry out individualized measures according to the gastrointestinal function of the patients, follow the principle of a small number of times, increments gradually, and closely observe the changes of the patient's condition.

\subsubsection{Postoperative pain management}

Effective postoperative analgesia is one of the important contents of ERAS. Pain control management is best implemented by anesthesiologist, pain specialist, surgeon and nurse. The necessary psychological guidance can reduce the use of painkillers and shorten the length of stay in hospital ${ }^{14}$. After the operation, the long-acting local anesthetic can be injected into the incision area, and the epidural catheter can be used for analgesia with opioid drugs. Which have positive effect on adverse reaction, and decrease the influence on the respiratory tract and gastrointestinal function. There is a great analgesic effect of (NSAIDs) and COX2 blockers combined with peripheral non-steroidal anti-inflammatory drugs. However, traditional opioids can lead to some adverse reactions, such as nausea, respiratory depression, gastrointestinal function recovery and addiction. The study found that compared with systemic intravenous opioid analgesics, epidural analgesia can significantly reduce the risk of postoperative death, and the incidence of atrial fibrillation, supraventricular tachycardia, deep venous thrombosis, respiratory inhibition, atelectasis, pneumonia, intestinal obstruction and postoperative nausea and vomiting, and promote the recovery of intestinal function. However, there are hypotension, pruritus, urinary retention and motor nerve block problems ${ }^{15}$.

\subsubsection{Management of postoperative complications}

The incidence of postoperative complications of gastric cancer is $10-40 \%$. Serious complications can lead to death, increase the incidence of unplanned reoperation and readmission, prolong hospitalization time, increase economic burden, and seriously affect the prognosis of patients. The changes of vital signs should be observed closely after operation. In case of serious complications, doctors should follow the " $3 \mathrm{~s}$ " principle. That is, to take active measures to maintain the sustention of basic vital signs. To support the patient's visceral function and supplement various substances and nutrients. the " $3 \mathrm{~s}$ " principle can be highly summed up the core concept of treatment during the successful treatment of many critical gastrointestinal patients.

\section{Multidisciplinary Cooperation Management in the Development of ERAS \\ 2.1 Multidisciplinary teams}

The patient is an organic whole, may be combined with a variety of diseases or needs a variety of treatment methods, which requires multi-department collaboration treatment. ERAS has been popularized and applied in various surgical disciplines. Its main contents include patient education, optimization of anesthetic methods, reduction of surgical stress, reduction of postoperative nausea, vomiting and intestinal paralysis, full analgesia, and rational use of various drainage tubes. Early nutrition and activities out of bed, standardized discharge standards, etc. This also means that the clinical development of ERAS necessarily involves anesthesia, surgery, nursing, nutrition and other related departments.

For a long time, it has been regarded that surgeons play the main role in the treatment of gastrointestinal neoplasms. However, modern oncology has put forward new and higher requirements for surgeons. The treatment of modern gastrointestinal tumors involve medical imaging department, oncology department, radiotherapy department, pathology department, anesthesiology department, surgery and so on, then the MDT model emerges as the times require. The work of MDT includes preoperative staging (professional radiographs and endoscopes), pathological staging, evaluation of neoadjuvant chemoradiotherapy efficacy and targeting site detection, and postoperative comprehensive treatment. If the relative discipline personnel can be relatively fixed and specialized, it can also provide a great practical basis for the standardization of gastrointestinal surgery. On this basis, it can provide individualized treatment for patients and improve the long-term outcome.

\subsection{Change of Multidisciplinary concept}

ERAS clinical studies show that the goal of accelerating the recovery of patients cannot be achieved by simply changing the techniques or methods. At present, it has gained consensus on the multidisciplinary collaboration of ERAS. But due to the independence of various professional fields, it is still very difficult to realize the coordination and unification of clinical subjects. The current ERAS program mainly consists of surgeons, anesthesia and other related disciplines involved in the whole process of treatment. Although there have been great achievements, but still shortcomings, which are highlighted by the lack of coordination between the program makers and the practitioners of the relevant procedures. Because of the difference of patients' situation, anesthesiologists and the surgeons, there's also a big diversity in the effect of execution. Surgery is difficult to make accurate evaluation on anesthetic methods and effects, and surgery cannot give professional guidance to anesthetic work. Vice versa, 
anesthesiology is an indirect management of patients, unable to determine the operation mode and postoperative rehabilitation management. It is obvious that the single subject-led ERAS program is difficult to effectively integrate the related disciplines and professional habits. Therefore, the effective promotion of ERAS requires multidisciplinary conceptual change and renewal. The key, to improve the understanding of the connotation of ERAS, is to break through the "barrier" effect of discipline, and to adhere to the deep integration of multi-disciplinary teams under the principle of patient-centered, especially the coordination of anesthesia and surgery ${ }^{17}$.

First of all, the principle of ERAS is patient-centered, and all relevant disciplines implement effective methods of diagnosis and treatment around the interests of patients. Secondly, the therapeutic effect should be evaluated by relevant professional departments, for example, anaesthesiology evaluate anesthesia and analgesic effect, prevention and treatment of postoperative nausea and vomiting and cognitive impairment, and so on. Third, multidisciplinary collaborative ERAS therapy requires an overall organizer, such as the top-level designer of the hospital, whose role is to effectively organize collaborative relationships in related disciplines. Coordinate the professional cooperation between disciplines to work out the overall implementation plan of ERAS. However, no matter what kind of modes ares adopted, the patient's benefit should be the center, in order to promote the patient's early recovery and to realize the maximum satisfaction of the patient.

\subsection{ERAS has only the beginning, not the end}

In 1997, the concept of ERAS was proposed Professor Henrik Kelhet in Denmark, the core of which is to reduce trauma and stress. In each stage of perioperative period, the psychological and physical stress response of the patients was reduced through various measures. The aim is to shorten the hospital stay, reduce the incidence of complications, reduce the risk of readmission, reduce mortality and reduce medical costs. Reviewing years of practical research on ERAS, Professor Kehlet believes that effective implementation of ERAS requires multidisciplinary collaboration ${ }^{18,19}$, and that the study of ERAS must adhere to its original intention to perform its duties in various professional disciplines around the theme of patient-centred, and to realize the early recovery of the patients on the basis of safety and painless ${ }^{20}$. Therefore, it is not difficult to understand that the core of ERAS is to focus on patients and fully fulfill their respective responsibilities. ERAS is only a comprehensive idea about the interests of patients from the overall point of view.

From the perspective of development, ERAS is also evolving, from "fast-track surgery", which started with optimizing the surgical management process, to ERAS, which focuses on every aspect of the perioperative period. However, no matter how it develops, patient-centered will be the eternal core of ERAS. With the transformation and integration of modern medicine, the connotation of ERAS will be expanded to meet the increasing needs of patients, from improving the operation transformed into improving the humanities, from procedural, standardized to individualized.
Human medicine needs are growing, ERAS will continue to improve, a long way to go, endless development ${ }^{17}$.

\section{Reference}

1. Mortensen K, Nilsson M, Slim K, et al. Consensus guidelines for enhanced recovery after gastrectomy: Enhanced Recovery After Surgery (ERAS(R)) Society recommendations. Br J Surg 2014; 101(10):1209-29.

2. Kehlet H, Wilmore DW. Multimodal strategies to improve surgical outcome. Am J Surg 2002; 183(6):630-41.

3. Visioni A, Shah R, Gabriel E, et al. Enhanced Recovery After Surgery for Noncolorectal Surgery?: A Systematic Review and Meta-analysis of Major Abdominal Surgery. Ann Surg 2018; 267(1):57-65.

4. Lassen $\mathrm{K}$, Ljungqvist $\mathrm{O}$, Dejong $\mathrm{CH}$, et al. Pancreaticoduodenectomy: ERAS recommendations. Clin Nutr 2013; 32(5):870-1.

5. Gianotti L, Biffi R, Sandini $M$, et al. Preoperative Oral Carbohydrate Load Versus Placebo in Major Elective Abdominal Surgery (PROCY): A Randomized, Placebo-controlled, Multicenter, Phase III Trial. Ann Surg 2018; 267(4):623-630.

6. Haynes AB, Weiser TG, Berry WR, et al. A surgical safety checklist to reduce morbidity and mortality in a global population. N Engl J Med 2009; 360(5):491-9.

7. Kehlet H. Postoperative pain, analgesia, and recovery-bedfellows that cannot be ignored. Pain 2018; 159 Suppl 1:S11-S16.

8. Makaryus R, Miller TE, Gan TJ. Current concepts of fluid management in enhanced recovery pathways. $\mathrm{Br} \mathrm{J}$ Anaesth 2018; 120(2):376-383.

9. Scott MJ, Baldini G, Fearon KC, et al. Enhanced Recovery After Surgery (ERAS) for gastrointestinal surgery, part 1: pathophysiological considerations. Acta Anaesthesiol Scand 2015; 59(10):1212-31.

10. Tohme S, Simmons RL, Tsung A. Surgery for Cancer: A Trigger for Metastases. Cancer Res 2017; 77(7):1548-1552.

11. Kim KH, Kim SH, Kim MC. How much progress has been made in minimally invasive surgery for gastric cancer in Korea?: a viewpoint from Korean prospective clinical trials. Medicine (Baltimore) 2014; 93(28):e233.

12. Yang Z, Zheng Q, Wang Z. Meta-analysis of the need for nasogastric or nasojejunal decompression after gastrectomy for gastric cancer. Br J Surg 2008; 95(7):809-16.

13. Willcutts $\mathrm{KF}$, Chung MC, Erenberg CL, et al. Early Oral Feeding as Compared With Traditional Timing of Oral Feeding After Upper Gastrointestinal Surgery: A Systematic Review and Meta-analysis. Ann Surg 2016; 264(1):54-63.

14. Joshi GP, Kehlet H, Group PW. Guidelines for perioperative pain management: need for re-evaluation. Br J Anaesth 2017; 119(4):703-706.

15. Popping DM, Elia N, Van Aken HK, et al. Impact of epidural analgesia on mortality and morbidity after surgery: systematic review and meta-analysis of randomized controlled trials. Ann Surg 2014; 259(6):1056-67.

16. Khazaei S, Rezaeian S, Soheylizad M, et al. Global Incidence and Mortality Rates of Stomach Cancer and the Human Development Index: an Ecological Study. Asian Pac J Cancer Prev 2016; 17(4):1701-4.

17. Kehlet H. ERAS Implementation-Time To Move Forward. Ann Surg 2018; 267(6):998-999.

18. Kehlet H. Enhanced Recovery After Surgery (ERAS): good for now, but what about the future? Can J Anaesth 2015; 62(2):99-104.

19. Kehlet H, Joshi GP. Enhanced Recovery After Surgery: Current Controversies and Concerns. Anesth Analg 2017; 125(6):2154-2155.

20. Meissner W, Huygen F, Neugebauer EAM, et al. Management of acute pain in the postoperative setting: the importance of quality indicators. Curr Med Res Opin 2018; 34(1):187-196. 